TRANSACTIONS OF THE

AMERICAN MATHEMATICAL SOCIETY

Volume 364, Number 7, July 2012, Pages 3609-3626

S 0002-9947(2012)05472-9

Article electronically published on February 20, 2012

\title{
TOTALLY UMBILICAL HYPERSURFACES OF MANIFOLDS ADMITTING A UNIT KILLING FIELD
}

\author{
RABAH SOUAM AND JOERI VAN DER VEKEN
}

\begin{abstract}
We prove that a Riemannian product of type $\mathbb{M}^{n} \times \mathbb{R}$ admits totally umbilical hypersurfaces, which are neither horizontal nor vertical, if and only if $\mathbb{M}^{n}$ has locally the structure of a warped product and we give a complete description of the totally umbilical hypersurfaces in this case. Moreover, we give a necessary and sufficient condition under which a Riemannian threemanifold carrying a unit Killing field admits totally geodesic surfaces and we study local and global properties of three-manifolds satisfying this condition.
\end{abstract}

\section{INTRODUCTION}

The starting point of the research leading to this paper was the classification of totally umbilical surfaces in three-dimensional homogeneous spaces with a fourdimensional isometry group, which can be found in [11] and [12. As is well known, these three-spaces admit Riemannian submersions onto surfaces of constant Gaussian curvature and the unit vector field tangent to the fibers is Killing. It turns out that such a space admits totally umbilical surfaces if and only if it is a Riemannian product of the base surface and the fibers, i.e., if and only if its universal covering is either $\mathbb{S}^{2}(\kappa) \times \mathbb{R}$ or $\mathbb{H}^{2}(\kappa) \times \mathbb{R}$. Moreover, the obtained classification of totally umbilical surfaces was extended to a classification of totally umbilical hypersurfaces of the conformally flat symmetric manifolds $\mathbb{S}^{n}(\kappa) \times \mathbb{R}$ and $\mathbb{H}^{n}(\kappa) \times \mathbb{R}$ in [13] and 3.

Two questions for further generalizations come up naturally now.

(1) When does a Riemannian product of type $\mathbb{M}^{n} \times \mathbb{R}$ admit totally umbilical hypersurfaces and what are they?

(2) When does a Riemannian three-space with a unit Killing field admit totally umbilical surfaces and what are they?

In this paper, we give a complete answer to the first question. Our Theorem 1 states that a necessary and sufficient condition for $\mathbb{M}^{n} \times \mathbb{R}$ to admit totally umbilical hypersurfaces is that $\mathbb{M}^{n}$ itself has (locally) the structure of a warped product. Moreover, we give a full description of all totally umbilical hypersurfaces of such a manifold and we remark that our results are still valid if we start with a warped product instead of with a Riemannian product as ambient space.

Received by the editors June 29, 2010.

2010 Mathematics Subject Classification. Primary 53B25, 53C40, 53C42.

Key words and phrases. Totally umbilical, totally geodesic, product manifold, Killing field, warped product.

The second author is a post-doctoral researcher supported by the Research Foundation, Flanders (F.W.O.).

This work was done while the second author visited the Université Paris Diderot, Paris 7 supported by a grant of the Research Foundation, Flanders (F.W.O.).

(C)2012 American Mathematical Society Reverts to public domain 28 years from publication 
For the second question we give a partial answer. We find a necessary and sufficient condition for a three-manifold with a unit Killing field to admit totally geodesic surfaces. We remark that it is not necessary that the three-manifold reduces to a Riemannian product, a fact which is already illustrated by the standard three-sphere. We describe the totally geodesic surfaces and we study the local and global properties of three-spaces satisfying our condition.

We are grateful to Eric Toubiana for valuable remarks on a first version of this work.

\section{Preliminaries}

Let $\left(M^{n}, g\right) \rightarrow\left(\tilde{M}^{n+1}, \tilde{g}\right)$ be an isometric immersion between Riemannian manifolds. If $N$ is a unit normal vector field along the immersion and $\nabla$ and $\tilde{\nabla}$ are the Levi-Civita connections of $\left(M^{n}, g\right)$ and $\left(\tilde{M}^{n+1}, \tilde{g}\right)$, then the second fundamental form $h$ and the shape operator $S$ associated to $N$ are defined by the formulas of Gauss and Weingarten: for any vector fields $X, Y$ on $M^{n}$ one has

$$
\tilde{\nabla}_{X} Y=\nabla_{X} Y+h(X, Y) N, \quad S X=-\tilde{\nabla}_{X} N
$$

It is easy to check that $S$ is a symmetric $(1,1)$-tensor field on $M^{n}$, which is related to $h$ by $h(X, Y)=g(S X, Y)$. We call the immersion totally umbilical if $S$ is a multiple of the identity at every point and we call it totally geodesic if $S$ vanishes identically.

In our results, some special types of vector fields on Riemannian manifolds will occur. Let $(M, g)$ be a Riemannian manifold and let $\xi$ be a vector field on $M$. Then $\xi$ is said to be Killing if and only if $\mathcal{L}_{\xi} g=0$, where $\mathcal{L}$ is the Lie derivative. This condition means that the flow of $\xi$ consists of isometries, and in terms of the Levi-Civita connection $\nabla$, one can reformulate it as

$$
g\left(\nabla_{X} \xi, Y\right)+g\left(\nabla_{Y} \xi, X\right)=0
$$

for all $p \in M$ and $X, Y \in T_{p} M$.

More generally, $\xi$ is said to be conformal if and only if $\mathcal{L}_{\xi} g=2 \phi g$ for some function $\phi$. This means that the flow of $\xi$ consists of conformal maps.

Finally, we say that $\xi$ is closed conformal if and only if it is conformal and its dual one-form is closed. It can be checked by a straightforward computation that $\xi$ is closed conformal if and only if

$$
\nabla_{X} \xi=\phi X
$$

for all $p \in M$ and all $X \in T_{p} M$, where $\phi$ is as above.

In all of what follows the manifolds will be assumed of class $C^{\infty}$.

\section{Totally Umbilical hypersurfaces of MANifolds of TYPE $\mathbb{M}^{n} \times I$}

Denote by $\mathbb{M}^{n} \times I$ the Riemannian product of a Riemannian manifold $\left(\mathbb{M}^{n}, g_{\mathbb{M}^{n}}\right)$ and an open interval $I$ of the Euclidean line and let $\pi: \mathbb{M}^{n} \times I \rightarrow \mathbb{M}^{n}$ be the canonical projection. We shall denote by $\xi$ a unit vector field on $\mathbb{M}^{n} \times I$, tangent to the fibers of $\pi$. We remark that $\xi$ is a unit Killing field.

There are two natural families of examples of totally geodesic hypersurfaces of $\mathbb{M}^{n} \times I$, namely the slices $\mathbb{M}^{n} \times\left\{t_{0}\right\}, t_{0} \in I$ and the inverse images under $\pi$ of totally geodesic hypersurfaces of $\mathbb{M}^{n}$, if they exist. We are thus interested in totally umbilical hypersurfaces which are at some point neither orthogonal nor tangent to $\xi$. 
If $\Sigma$ is a hypersurface of $\mathbb{M}^{n} \times I$ with unit normal $N$, one can define a vector field $T$ and a real-valued function $\nu$ on $\Sigma$ by the following orthogonal decomposition of $\xi:$

$$
\xi=T+\nu N
$$

Then $T$ and $\nu$ satisfy the following equations.

Lemma 1. Let $\Sigma$ be a hypersurface in $\mathbb{M}^{n} \times I$ and denote by $\nabla$ the Levi-Civita connection of $\Sigma$, by $S$ the shape operator of the immersion and by $h$ the second fundamental form. Then for any vector $X$ tangent to $\Sigma$,

$$
\nabla_{X} T=\nu S X, \quad X(\nu)=-h(X, T) .
$$

Proof. Denote by $\widetilde{\nabla}$ the Levi-Civita connection of $\mathbb{M}^{n} \times I$. Since $\xi$ is a parallel vector field on $\mathbb{M}^{n} \times I$, one has $\widetilde{\nabla}_{X} \xi=0$. Now let $X$ be tangent to $\Sigma$. By the definitions of $T$ and $\nu$ and by using the formulas of Gauss and Weingarten, it follows that

$$
0=\tilde{\nabla}_{X} \xi=\tilde{\nabla}_{X}(T+\nu N)=\nabla_{X} T+h(X, T) N+X(\nu) N-\nu S X
$$

The result follows by considering the tangent (resp. normal) component of the above equation.

If $\Sigma$ is totally umbilical in $\mathbb{M}^{n} \times I$, say $S=\lambda$ id, then $T$ is a closed conformal field on $\Sigma$. Indeed, in this case it follows from Lemma 1 above that $\nabla_{X} T=\nu \lambda X$ for all vector fields $X$ tangent to $\Sigma$. We shall now prove that if $\Sigma$ is non-vertical and non-horizontal at some point $p$, we can use $T$ to construct a local non-vanishing conformal field on $\mathbb{M}^{n}$.

Proposition 1. Let $\Sigma$ be a totally umbilical hypersurface of $\mathbb{M}^{n} \times I$, which is neither vertical nor horizontal at some point. Then the canonical projection $\pi: \mathbb{M}^{n} \times I \rightarrow$ $\mathbb{M}^{n}$ is locally a diffeomorphism between an open neighborhood $U$ of this point in $\Sigma$ and the open subset $\pi U$ of $\mathbb{M}^{n}$. Let $T$ be as above and denote by $T_{0}$ the projection of $T$ to $\pi U$, rescaled such that it has the same length as $T$ again. Then $T_{0}$ is a closed conformal field on $\pi U$.

Proof. Let $\Sigma$ be a totally umbilical hypersurface of $\mathbb{M}^{n} \times I$. Let $\xi, N, T$ and $\nu$ be as above and assume that the shape operator associated to $N$ is $S=\lambda$ id. Suppose $\Sigma$ is non-vertical and non-horizontal at some point, then it is clear that there is an open neighborhood $U$ of this point in $\Sigma$ where $\nu$ does not vanish and such that $\pi$ is a local diffeomorphism between $U$ and its image $\pi U$ in $\mathbb{M}^{n}$. First, extend the vector fields $T, N$ and the functions $\nu, \lambda$ to the whole of $\pi U \times I$ by using the one-parameter group of translations corresponding to the Killing field $\xi$ and denote these again by $T, N, \nu$ and $\lambda$. Since $\nu$ and $\lambda$ are constant on fibers of $\pi$, one can also view them as functions on $\pi U$. Using this notation, the vector field $T_{0}$ on $\pi U$ is

$$
T_{0}=(d \pi)(T) \frac{\|T\|}{\|(d \pi)(T)\|}=\frac{1}{\nu}(d \pi)(T)
$$

and its horizontal lift to $\pi U \times I$ is

$$
\widetilde{T_{0}}=\nu \xi-N=\frac{\nu^{2}-1}{\nu} \xi+\frac{1}{\nu} T
$$

We remark that $T_{0}$ is, up to the sign, the projection of $N$ to $\pi U$. 
Now let $X$ be a vector field on $\pi U$ and denote by $\widetilde{X}$ its horizontal lift. Then

$$
\nabla_{X}^{\mathbb{M}^{n}} T_{0}=(d \pi)\left(\widetilde{\nabla}_{\widetilde{X}} \widetilde{T}_{0}\right)=(d \pi)\left(\widetilde{\nabla}_{\widetilde{X}}(\nu \xi-N)\right)=-(d \pi)\left(\widetilde{\nabla}_{\widetilde{X}} N\right) .
$$

Let $Y$ be a local vector field on $\Sigma$ such that $(d \pi)(Y)=X$. Denote the extension to $\pi U \times I$, using the flow of $\xi$, again by $Y$. Then $\widetilde{X}=Y-\langle Y, \xi\rangle \xi$ and

$$
\widetilde{\nabla}_{\widetilde{X}} N=\widetilde{\nabla}_{Y-\langle Y, \xi\rangle \xi} N=\widetilde{\nabla}_{Y} N-\langle Y, \xi\rangle \widetilde{\nabla}_{\xi} N=-\lambda Y .
$$

Here we used that $[\xi, N]=0$ implies $\widetilde{\nabla}_{\xi} N=\widetilde{\nabla}_{N} \xi=0$. From (22) and (3), we obtain $\nabla_{X}^{\mathbb{M}^{n}} T_{0}=\lambda X$, which proves that $T_{0}$ is indeed closed conformal.

The fact that $\mathbb{M}^{n}$ admits a local closed conformal field determines locally its Riemannian structure, as shown by the following known result (see [6, 9] and the references therein).

Proposition 2. Let $V$ be a local closed conformal field without zeros on a Riemannian manifold $\mathbb{M}^{n}$, say $\nabla_{X}^{\mathbb{M}^{n}} V=f X$ for some function $f$ and for all vector fields $X$ on $\mathbb{M}^{n}$. Then $\mathbb{M}^{n}$ has locally the structure of a warped product of an interval of the Euclidean line with some $(n-1)$-dimensional Riemannian manifold.

Proof. One can check that the distribution orthogonal to $V$ is integrable and hence one can find a local coordinate system $\left(x_{1}, \ldots, x_{n}\right)$ on $\mathbb{M}^{n}$ such that $\partial_{x_{1}}=V$ and $\partial_{x_{j}}$ is orthogonal to $\partial_{x_{1}}$ for $j \geq 2$. With respect to these coordinates, the metric on $\mathbb{M}^{n}$ takes the form

$$
g=g_{11}\left(x_{1}, \ldots, x_{n}\right) d x_{1}^{2}+\sum_{i, j=2}^{n} g_{i j}\left(x_{1}, \ldots, x_{n}\right) d x_{i} d x_{j} .
$$

It follows from a straightforward computation that $\partial_{x_{j}} g_{11}=0$ for $j \geq 2$ and that $\partial_{x_{1}} g_{i j}=2 f g_{i j}$ for $i, j \geq 2$. Hence, one has

$$
g=g_{11}\left(x_{1}\right) d x_{1}^{2}+\exp \left(2 \int f d x_{1}\right) \sum_{i, j=2}^{n} c_{i j}\left(x_{2}, \ldots, x_{n}\right) d x_{i} d x_{j} .
$$

To conclude, we prove that $\partial_{x_{j}} f=0$ for $j \geq 2$, such that, after a change of the $x_{1}$-coordinate, the metric above is indeed a warped product metric. To see this, let $R$ be the curvature tensor of $\mathbb{M}^{n}$, then

$$
\begin{aligned}
0 & =\left\langle R\left(\partial_{x_{1}}, \partial_{x_{j}}\right) \partial_{x_{1}}, \partial_{x_{1}}\right\rangle=\left\langle\nabla_{\partial_{x_{1}}}^{\mathbb{M}^{n}} \nabla_{\partial_{x_{j}}}^{\mathbb{M}^{n}} \partial_{x_{1}}-\nabla_{\partial_{x_{j}}}^{\mathbb{M}^{n}} \nabla_{\partial_{x_{1}}}^{\mathbb{M}^{n}} \partial_{x_{1}}, \partial_{x_{1}}\right\rangle \\
& =\left\langle\left(\partial_{x_{1}} f\right) \partial_{x_{j}}-\left(\partial_{x_{j}} f\right) \partial_{x_{1}}, \partial_{x_{1}}\right\rangle=-\left(\partial_{x_{j}} f\right) g_{11}
\end{aligned}
$$

Remark 1 . The converse to Proposition 2 is also true. In a warped product $I \times{ }_{f} M=$ $\left(I \times M, d t^{2}+f(t)^{2} g_{M}\right)$, the field $f(t) \partial_{t}$ is closed conformal and vanishes nowhere.

We can now prove our main result in this section.

Theorem 1. A Riemannian product space $\mathbb{M}^{n} \times I$ admits a totally umbilical hypersurface $\Sigma$, which is neither vertical nor horizontal at some point $(p, t) \in \mathbb{M}^{n} \times I$, if and only if $\mathbb{M}^{n}$ has in a neighborhood of $p$ the structure of a warped product of an interval of the Euclidean line with some $(n-1)$-dimensional Riemannian manifold.

In particular, when $n=2$, there exists a totally umbilical surface in $\mathbb{M}^{2} \times I$, which is neither vertical nor horizontal at some point $(p, t) \in \mathbb{M}^{2} \times I$, if and only if $\mathbb{M}^{2}$ admits a Killing field in a neighborhood of $p$ which does not vanish at $p$. Moreover 
any such surface is invariant by a local one-parameter group of local isometries of $\mathbb{M}^{2} \times I$ keeping the factor I pointwise fixed.

Proof. It follows from Propositions 1 and 2 above that $\mathbb{M}^{n}$ having the structure of a warped product in a neighborhood of $p$ is a necessary condition for $\mathbb{M}^{n} \times I$ to admit a totally umbilical hypersurface which is non-vertical and non-horizontal at $(p, t)$.

We shall now prove that this condition is also sufficient. Assume that $\mathbb{M}^{n}=$ $J \times_{f} M^{n-1}$, i.e., that the metric on $\mathbb{M}^{n}$ can be written as

$$
g_{\mathbb{M}^{n}}=d x_{1}^{2}+f\left(x_{1}\right)^{2} g_{M^{n-1}}\left(x_{2}, \ldots, x_{n}\right) .
$$

Then the metric on $\mathbb{M}^{n} \times I$ can be written as

$$
g=d x_{0}^{2}+d x_{1}^{2}+f\left(x_{1}\right)^{2} g_{M^{n-1}}\left(x_{2}, \ldots, x_{n}\right) .
$$

We know from above that a non-vertical and non-horizontal totally umbilical hypersurface $\Sigma$ of $\mathbb{M}^{n} \times I$ should be tangent to the distribution orthogonal to the vector fields $\partial_{x_{0}}$ and $\partial_{x_{1}}$ at any of its points. This means that $\Sigma$ is generated by a curve in the $\left(x_{0}, x_{1}\right)$-plane, say $\alpha(s)=\left(x_{0}(s), x_{1}(s)\right)$. Assume that $\alpha$ is parametrized by arc length, then there exists a function $\theta$ such that

$$
x_{0}^{\prime}(s)=\sin \theta(s), \quad x_{1}^{\prime}(s)=\cos \theta(s) .
$$

In this case, the tangent space to $\Sigma$ is spanned by $X_{1}=\sin \theta(s) \partial_{x_{0}}+\cos \theta(s) \partial_{x_{1}}$, $X_{2}=\partial_{x_{2}}, \ldots, X_{n}=\partial_{x_{n}}$ and a unit normal to $\Sigma$ is given by $N=\cos \theta(s) \partial_{x_{0}}-$ $\sin \theta(s) \partial_{x_{1}}$.

One can compute the Levi-Civita connection $\widetilde{\nabla}$ of $\mathbb{M}^{n} \times I$ from the metric above to verify

$$
\widetilde{\nabla}_{X_{1}} N=-\theta^{\prime}(s) X_{1}, \quad \widetilde{\nabla}_{X_{j}} N=-\sin \theta(s) \frac{f^{\prime}}{f} X_{j}
$$

for every $j \geq 2$. Hence, $\Sigma$ is totally umbilical if and only if

$$
\theta^{\prime}(s)=\sin \theta(s) \frac{f^{\prime}}{f} .
$$

One can now use this equation to determine the functions $x_{0}(s)$ and $x_{1}(s)$. Indeed, we have

$$
x_{1}^{\prime \prime}(s)=-\sin \theta(s) \theta^{\prime}(s)=-\sin ^{2} \theta(s) \frac{f^{\prime}\left(x_{1}(s)\right)}{f\left(x_{1}(s)\right)}=-\left(1-x_{1}^{\prime}(s)^{2}\right) \frac{f^{\prime}\left(x_{1}(s)\right)}{f\left(x_{1}(s)\right)},
$$

which yields after a first integration

$$
x_{1}^{\prime}(s)= \pm \sqrt{1-c^{2} f\left(x_{1}(s)\right)^{2}}
$$

for some real constant $c$. This ODE for $x_{1}(s)$ is, at least locally, always solvable. The function $x_{0}(s)$ is then determined by

$$
x_{0}(s)=\int \sqrt{1-x_{1}^{\prime}(s)^{2}} d s=\int c f\left(x_{1}(s)\right) d s .
$$

Thus there does always exist a non-vertical and non-horizontal totally umbilical hypersurface of $\mathbb{M}^{n} \times I$ if $\mathbb{M}^{n}$ is locally isometric to the warped product described above. A parametrization for such a totally umbilical hypersurface $\Sigma$ is $\varphi\left(s, u_{1}, \ldots, u_{n-1}\right)=\left(x_{0}(s), x_{1}(s), u_{1}, \ldots, u_{n-1}\right)$. 
In the particular case when $n=2$, observe the following general fact that can be checked straightforwardly. Let $J$ denote the rotation over 90 degrees of an oriented Riemannian surface $M^{2}$, which is locally well defined on any Riemannian surface $M^{2}$. Then a vector field $X$ on $M^{2}$ is closed conformal if and only if $J X$ is Killing. Hence, if $\Sigma$ is a totally umbilical surface in $\mathbb{M}^{2} \times I$, then $J T$ is a Killing field on $\Sigma$. Moreover, $J T$ is orthogonal to the fibers of $\pi$ and $(d \pi)(J T)=J T_{0}$ is a Killing field on $\mathbb{M}^{2}$. This implies the result.

In particular, from Theorem 11 we recover the classification of totally umbilic surfaces in $\mathbb{S}^{2} \times \mathbb{R}$ and $\mathbb{H}^{2} \times \mathbb{R}$ obtained in [1] and [12].

Remark 2. Theorem 1 also gives a classification of totally umbilical hypersurfaces in Riemannian product spaces of type $\mathbb{M}^{n} \times I$. In particular, it shows that there is a totally geodesic hypersurface which is neither horizontal nor vertical at a point $(p, t) \in \mathbb{M}^{n} \times I$ if and only if $\mathbb{M}^{n}$ has, in a neighborhood of $p$, the structure of a Riemannian product $W \times J$ of some $(n-1)$-dimensional manifold $W$ with an interval $J$ of the Euclidean line. The totally geodesic hypersurfaces corresponding to this splitting, which are given in the proof of Theorem 1, are of the form $W \times L$ where $L$ is any geodesic in the flat factor $I \times J$.

Remark 3. It is interesting to observe that the results above provide a new proof for the classification of totally umbilic hypersurfaces in the Euclidean space $\mathbb{R}^{n+1}$ since the latter can be viewed (in various ways) as a product $\mathbb{R}^{n} \times \mathbb{R}$. The present proof has the advantage to work assuming only a $C^{2}$ regularity for the hypersurfaces. It can indeed easily be checked that $C^{2}$ regularity for the hypersurfaces is enough in the above results. The standard proof of the classification of totally umbilic hypersurfaces in $\mathbb{R}^{n+1}$ works for hypersurfaces with at least $C^{3}$ regularity. It is known this classification also holds for $C^{2}$-hypersurfaces (see [7, 10]).

Corollary 1. A Riemannian warped product space $I \times_{f} \mathbb{M}^{n}$ admits a totally umbilical hypersurface $\Sigma$, which is neither vertical nor horizontal at some point $(t, p) \in$ $I \times{ }_{f} \mathbb{M}^{n}$, if and only if $\mathbb{M}^{n}$ has in a neighborhood of $p$ the structure of a warped product of an interval of the Euclidean line with some $(n-1)$-dimensional Riemannian manifold.

Proof. First observe that a Riemannian warped product space $I \times{ }_{f} \mathbb{M}^{n}$ is conformal to a Riemannian product $J \times \mathbb{M}^{n}$, for some open interval $J$ of $\mathbb{R}$. Indeed, with some abuse of notation, the metric on $I \times_{f} \mathbb{M}^{n}$ writes

$$
g=d t^{2}+f(t)^{2} g_{\mathbb{M}^{n}}=f(t)^{2}\left(\frac{d t^{2}}{f(t)^{2}}+g_{\mathbb{M}^{n}}\right)
$$

Choosing a new parameter $s=\psi(t)=\int \frac{d t}{f(t)}$ and introducing the function $h(s)=$ $f\left(\psi^{-1}(s)\right)$, we can write

$$
g=h(s)^{2}\left(d s^{2}+g_{\mathbb{M}^{n}}\right)
$$

as desired.

Now the claim follows from Theorem 1 and the known fact that totally umbilic hypersurfaces are preserved under conformal diffeomorphisms between the ambient manifolds. 


\section{Totally geodesic surfaces in a three-Dimensional SPACE ADMitTing A UNIT KILLING FIELD}

In this section we will characterize locally the Riemannian three-manifolds admitting a unit Killing field which possess totally geodesic surfaces. A good reference on manifolds admitting a Killing field of constant length is given by [1].

We start with a result which is valid in all dimensions. Let $M$ denote a Riemannian manifold which admits a unit Killing field $\xi$. The product manifolds $\mathbb{M}^{n} \times I$ considered in Section 3 are a particular case. Denote by $\widetilde{\nabla}$ the Levi-Civita connection of $M$. Let $\Sigma$ be a hypersurface in $M$ with unit normal $N$. Then we can, as in the previous section, define a vector field $T$ and a real-valued function $\nu$ on $\Sigma$ by the orthogonal decomposition

$$
\xi=T+\nu N
$$

The following result is a key fact for our purposes:

Proposition 3. Let $\Sigma$ be a totally geodesic hypersurface in a Riemannian manifold $M$ admitting a unit Killing field $\xi$. Suppose $\xi$ is not tangent to $\Sigma$ at some point. Then one can extend the vector field $T$ to a neighborhood of this point in $M$ using the local flow of $\xi$. If one denotes the resulting vector field again by $T$, then $T$ is a local Killing field on $M$.

Proof. Since $\xi$ is transversal to $\Sigma$ in a neighborhood of the point, using the local flow $\left(\phi_{t}\right)_{t \in I}$ of $\xi$, we obtain a foliation $\mathcal{F}$ of an open subset of $M$ by the totally geodesic hypersurfaces $\phi_{t}(\Sigma)$. In this way we have local extensions of the fields $T$ and $N$ and the function $\nu$. Denote these extensions again by $T, N$ and $\nu$. Note that (4) is again valid for these extensions.

We have to verify that $\left\langle\widetilde{\nabla}_{X} T, Y\right\rangle+\left\langle X, \widetilde{\nabla}_{Y} T\right\rangle=0$ for all vector fields $X$ and $Y$. First we note that

$$
T(\nu)=0 .
$$

Indeed, since the hypersurfaces $\phi_{t}(\Sigma)$ are totally geodesic and $\xi$ is a unit Killing field, we have

$$
T(\nu)=T(\langle\xi, N\rangle)=\left\langle\widetilde{\nabla}_{T} \xi, N\right\rangle=\left\langle\widetilde{\nabla}_{T} \xi, \frac{1}{\nu}(T-\xi)\right\rangle=0 .
$$

Now consider a vector field $X$ which is tangent to the leaves of the foliation $\mathcal{F}$. Then

$$
\widetilde{\nabla}_{X} T=\widetilde{\nabla}_{X} \xi-X(\nu) N
$$

Furthermore,

$$
\widetilde{\nabla}_{N} T=\widetilde{\nabla}_{\frac{1}{\nu}(\xi-T)} T=\frac{1}{\nu}\left(\widetilde{\nabla}_{T} \xi-\widetilde{\nabla}_{T} T\right) .
$$

Using (6) and (5) we get

$$
\widetilde{\nabla}_{N} T=0
$$

Using (6) and (7), it is easy to check that $T$ is Killing.

We now particularize to the case where the ambient manifold is three-dimensional. Well-known examples of such manifolds are Riemannian products of type $\mathbb{M}^{2} \times \mathbb{R}$ and also the unit three-sphere $\mathbb{S}^{3}$, Berger spheres and the Thurston spaces 
$\widetilde{\mathrm{SL}}(2, \mathbb{R})$ and $\mathrm{Nil}_{3}$. In the next section we will describe more such spaces. We first prove an important basic formula.

Lemma 2. Let $M^{3}$ be an oriented Riemannian manifold carrying a unit Killing field $\xi$. Denote by $\widetilde{\nabla}$ the Levi-Civita connection of $M^{3}$ and by $\times$ its cross product. Then there exists a real-valued function $\tau$ on $M^{3}$, with $\xi(\tau)=0$, such that

$$
\widetilde{\nabla}_{X} \xi=\tau(X \times \xi)
$$

for all vector fields $X$ on $M^{3}$.

Proof. It is clear that $\widetilde{\nabla}_{X} \xi$ is perpendicular to $\xi$ and $X$ since $\xi$ is a unit Killing field. Because the space is three-dimensional, we obtain that $\widetilde{\nabla}_{X} \xi=\tau(X)(X \times \xi)$ for some real number $\tau(X)$.

Since the mapping $T_{p} M^{3} \rightarrow T_{p} M^{3}: X \mapsto \widetilde{\nabla}_{X} \xi=\tau(X)(X \times \xi)$ must be linear for every point $p$, it is easily seen that $\tau$ can only depend on the choice of $p \in M^{3}$ and not on the choice of $X \in T_{p} M^{3}$. Hence $\tau$ is a real-valued function on $M^{3}$.

To see that this function satisfies $\xi(\tau)=0$, let $\left(\phi_{t}\right)_{t \in I}$ be the local flow of $\xi$ as above. Then $\widetilde{\nabla}_{\left(d \phi_{t}\right) X}\left(d \phi_{t}\right) \xi=\left(d \phi_{t}\right)\left(\widetilde{\nabla}_{X} \xi\right)$, or, equivalently, $\tau\left(\phi_{t}(p)\right)\left(\left(d \phi_{t}\right) X \times \xi\right)=$ $\tau(p)\left(\left(d \phi_{t}\right) X \times \xi\right)$ for every parameter $t$ and for every $p \in M^{3}$ and $X \in T_{p} M^{3}$. We conclude that $\tau\left(\phi_{t}(p)\right)=\tau(p)$ and hence that $\xi(\tau)=0$.

The first main result in this section is the following.

Theorem 2. Let $M$ be a Riemannian three-manifold carrying a unit Killing field $\xi$ and $p$ a point in $M$. Then

(1) $M$ admits a totally geodesic surface passing through $p$ which is everywhere orthogonal to $\xi$ if and only if $M$ has in a neighborhood of $p$ a Riemannian product structure $\Sigma \times I$ of some surface $\Sigma$ with an interval $I$ and $\xi$ is tangent to the factor $I$.

(2) $M$ admits a totally geodesic surface passing through $p$ which is everywhere tangent to $\xi$ if and only if there exists a geodesic through $p$ in $M$ on which $\tau$ vanishes and which is orthogonal to $\xi$ at $p$.

(3) The following three assertions are equivalent.

(i) $M$ admits a totally geodesic surface passing through $p$ which is neither orthogonal nor tangent to $\xi$ at $p$.

(ii) There is in a neighborhood of $p$ in $M$ an orthogonal decomposition $\xi=X_{1}+X_{2}$ of $\xi$, where $X_{1}$ and $X_{2}$ are Killing fields without zeros that commute.

(iii) There exist local coordinates $(x, y, z)$ around $p$ in $M$ with $\xi=\partial_{y}+\partial_{z}$, such that the metric takes the form

$$
g=d x^{2}+\sin ^{2} \theta(x) d y^{2}+\cos ^{2} \theta(x) d z^{2} .
$$

Remark 4. Statement (1) is valid in all dimensions. In dimension 3 it is equivalent to the vanishing of $\tau$ in a neighborhood of $p$.

Proof. We denote by $\left(\phi_{t}\right)_{t \in I}$ the local flow of $\xi$.

(1) Suppose $M$ admits a totally geodesic surface $\Sigma$, passing through $p$, which is everywhere orthogonal to $\xi$. Restricting $\Sigma$ and the interval $I$ if necessary, the mapping $(x, t) \in \Sigma \times I \mapsto \phi_{t}(x) \in M$ parametrizes an open subset of $M$ and is easily checked to be an isometry between the Riemannian product manifold $\Sigma \times I$ and that open set. 
Conversely it is clear that if $M$ has locally a product structure of some surface $\Sigma$ and an interval $I$ to which $\xi$ is tangent, then the surfaces $\Sigma \times\{t\}$ are totally geodesic and orthogonal to $\xi$ at each point.

(2) Suppose $s \in J \mapsto \gamma(s)$ is a geodesic of $M$ parametrized by arc length such that $\gamma\left(t_{0}\right)=p$ and $\left\langle\gamma^{\prime}\left(t_{0}\right), \xi\right\rangle=0$ for some $t_{0} \in J$ and $\tau(\gamma(s))=0$ for all $s \in J$. Note first that $\gamma$ is everywhere orthogonal to $\xi$. Indeed, for all $s \in J$,

$$
\frac{d}{d s}\left\langle\gamma^{\prime}(s), \xi\right\rangle=\left\langle\frac{D \gamma^{\prime}}{d s}(s), \xi\right\rangle+\left\langle\gamma^{\prime}(s), \tau(\gamma(s))\left(\gamma^{\prime}(s) \times \xi\right)\right\rangle=0
$$

The mapping $(s, t) \mapsto \phi_{t}(\gamma(s))$ parametrizes a surface $\Sigma$ in $M$ and $\left.\tau\right|_{\Sigma}=0$.

We now check that $\Sigma$ is totally geodesic. A unit normal field to $\Sigma$ is the field $N(s, t)=\left(d \phi_{t}\right)\left(\gamma^{\prime}(s)\right) \times \xi$. Note that $\xi$ commutes with $\left(d \phi_{t}\right)\left(\gamma^{\prime}(s)\right)$, so that

$$
\widetilde{\nabla}_{\xi}\left(d \phi_{t}\right)\left(\gamma^{\prime}(s)\right)=\widetilde{\nabla}_{\left(d \phi_{t}\right)\left(\gamma^{\prime}(s)\right)} \xi=0,
$$

where we used that $\left.\tau\right|_{\Sigma}=0$. Therefore

$$
\widetilde{\nabla}_{\xi} N=\widetilde{\nabla}_{\xi}\left(d \phi_{t}\right)\left(\gamma^{\prime}(s)\right) \times \xi+\left(d \phi_{t}\right)\left(\gamma^{\prime}(s)\right) \times \widetilde{\nabla}_{\xi} \xi=0 .
$$

Since $s \mapsto \phi_{t}(\gamma(s))$ is a geodesic in $M$ for each $t$, we have

$$
\widetilde{\nabla}_{\left(d \phi_{t}\right)\left(\gamma^{\prime}(s)\right)} N=\widetilde{\nabla}_{\left(d \phi_{t}\right)\left(\gamma^{\prime}(s)\right)}\left(d \phi_{t}\right)\left(\gamma^{\prime}(s)\right) \times \xi+\left(d \phi_{t}\right)\left(\gamma^{\prime}(s)\right) \times \widetilde{\nabla}_{\left(d \phi_{t}\right)\left(\gamma^{\prime}(s)\right)} \xi=0 .
$$

It follows that $\Sigma$ is totally geodesic.

Conversely, suppose $M$ admits a totally geodesic surface $\Sigma$ passing through $p$ which is everywhere tangent to $\xi$. As geodesics on $\Sigma$ are also geodesics on $M$, it is enough to check that $\left.\tau\right|_{\Sigma}=0$. This is indeed the case: take an arbitrary point $q$ of $\Sigma$ and a vector $X$ tangent to $\Sigma$ and linearly independent of $\xi$. Since $\Sigma$ is totally geodesic, the vector $\widetilde{\nabla}_{X} \xi=\tau(q)(X \times \xi)$ has to be tangent to $\Sigma$, which is only possible if $\tau(q)=0$.

(3) First, we prove that (i) implies (iii). Let $\Sigma$ be totally geodesic in $M$ such that $\xi$ is not tangent to $\Sigma$ at $p$. Extend $T, N, J T=N \times T$ and $\nu$ to a neighborhood of this $p$ in $M$ using the local flow of $\xi$. Using equations (6), (5) and (4), we have

$$
\begin{aligned}
& \widetilde{\nabla}_{J T} T=\widetilde{\nabla}_{J T} \xi-(J T)(\nu) N=\tau[(J T \times \xi)-\langle J T \times \xi, N\rangle N]=\tau(J T \times \nu N)=\tau \nu T, \\
& \widetilde{\nabla}_{T} J T=\widetilde{\nabla}_{T}(N \times T)=N \times \widetilde{\nabla}_{T}(\xi-\nu N)=N \times \tau(T \times \xi)=\tau \nu T .
\end{aligned}
$$

It follows that

$$
\begin{aligned}
& {[T, J T]=\widetilde{\nabla}_{T} J T-\widetilde{\nabla}_{J T} T=0} \\
& {[T, \nu N]=[T, \xi]=0} \\
& {[J T, \nu N]=[J T, \xi]=0 .}
\end{aligned}
$$

Hence, we can take local coordinates $(x, y, z)$ on $M$ such that $\partial_{x}=J T, \partial_{y}=T$ and $\partial_{z}=\nu N$. With respect to these coordinates, the metric takes the form

$$
g=\left(1-\nu^{2}\right)\left(d x^{2}+d y^{2}\right)+\nu^{2} d z^{2}
$$

From (5) one has $\partial_{y} \nu=T(\nu)=0$ and $\partial_{z} \nu=\nu N(\nu)=(\xi-T)(\nu)=0$. After a change of the $x$-coordinate, we obtain the form for $g$ given in the theorem.

To see that (iii) implies (ii), it suffices to take $X_{1}=\partial_{y}$ and $X_{2}=\partial_{z}$.

It remains to prove that (ii) implies (i). Let $u$ be a unit vector field perpendicular to $X_{1}$ and $X_{2}$. We shall first prove that $u$ commutes with $X_{1}$ and $X_{2}$. By using 
that $u$ is perpendicular to $X_{1}$ and that $X_{1}$ is Killing, we obtain

$$
\begin{aligned}
\left\langle\left[X_{1}, u\right], X_{1}\right\rangle & =\left\langle\widetilde{\nabla}_{X_{1}} u, X_{1}\right\rangle-\left\langle\widetilde{\nabla}_{u} X_{1}, X_{1}\right\rangle \\
& =-\left\langle u, \widetilde{\nabla}_{X_{1}} X_{1}\right\rangle-\left\langle\widetilde{\nabla}_{u} X_{1}, X_{1}\right\rangle \\
& =0 .
\end{aligned}
$$

Furthermore, by using that $u$ is perpendicular to $X_{2}$ and that $X_{1}$ is Killing, we find

$$
\begin{aligned}
\left\langle\left[X_{1}, u\right], X_{2}\right\rangle & =\left\langle\widetilde{\nabla}_{X_{1}} u, X_{2}\right\rangle-\left\langle\widetilde{\nabla}_{u} X_{1}, X_{2}\right\rangle \\
& =-\left\langle u, \widetilde{\nabla}_{X_{1}} X_{2}\right\rangle+\left\langle\widetilde{\nabla}_{X_{2}} X_{1}, u\right\rangle \\
& =-\left\langle\left[X_{1}, X_{2}\right], u\right\rangle=0 .
\end{aligned}
$$

Finally, since $\|u\|=1$ and $X_{1}$ is Killing,

$$
\left\langle\left[X_{1}, u\right], u\right\rangle=\left\langle\widetilde{\nabla}_{X_{1}} u, u\right\rangle-\left\langle\widetilde{\nabla}_{u} X_{1}, u\right\rangle=0 .
$$

We conclude that $\left[X_{1}, u\right]=0$. Analogously, we can prove that $\left[X_{2}, u\right]=0$.

Now consider an integral surface of the distribution spanned by $u$ and $X_{1}$. Of course, this surface is nowhere tangent or orthogonal to $\xi=X_{1}+X_{2}$. We shall prove that it is totally geodesic. It is sufficient to verify that $\widetilde{\nabla}_{u} u, \widetilde{\nabla}_{u} X_{1}$ and $\widetilde{\nabla}_{X_{1}} X_{1}$ are all perpendicular to $X_{2}$. Using Koszul's formula and the facts that $\left[X_{1}, X_{2}\right]=\left[X_{1}, u\right]=\left[X_{2}, u\right]=0,\left\langle X_{1}, X_{2}\right\rangle=\left\langle X_{1}, u\right\rangle=\left\langle X_{2}, u\right\rangle=0$ and $\langle u, u\rangle=1$ gives immediately that $\left\langle\widetilde{\nabla}_{u} u, X_{2}\right\rangle=0$ and $\left\langle\widetilde{\nabla}_{u} X_{1}, X_{2}\right\rangle=0$. Finally, using the facts that $X_{1}$ and $X_{2}$ are orthogonal and that $X_{2}$ is Killing gives $\left\langle\widetilde{\nabla}_{X_{1}} X_{1}, X_{2}\right\rangle=$ $-\left\langle X_{1}, \widetilde{\nabla}_{X_{1}} X_{2}\right\rangle=0$.

Remark 5. For later use, we note that in the coordinates where the metric takes the given form in case (iii) we have from the proof of Theorem 2 that $\partial_{x}=J T /\|T\|$, $\partial_{y}=T, \cos \theta(x)=\langle\xi, N\rangle$ and $\|T\|=\sin \theta(x)$.

We now study case (3) in Theorem 2 further. We are able to determine all the totally geodesic surfaces of $M$ in a neighborhood of $p$ in this case. We will need the following result which can be verified by straightforward computations.

Proposition 4. The Levi-Civita connection of the metric $g$ defined in local coordinates $(x, y, z)$ by

$$
g=d x^{2}+\sin ^{2} \theta(x) d y^{2}+\cos ^{2} \theta(x) d z^{2}
$$

is given by

$$
\begin{aligned}
& \widetilde{\nabla}_{\partial_{x}} \partial_{x}=0 \\
& \widetilde{\nabla}_{\partial_{x}} \partial_{y}=\cot \theta \theta^{\prime} \partial_{y}, \quad \widetilde{\nabla}_{\partial_{x}} \partial_{z}=-\tan \theta \theta^{\prime} \partial_{z}, \\
& \widetilde{\nabla}_{\partial_{y}} \partial_{y}=-\cos \theta \sin \theta \theta^{\prime} \partial_{x}, \quad \widetilde{\nabla}_{\partial_{y}} \partial_{z}=0, \quad \widetilde{\nabla}_{\partial_{z}} \partial_{z}=\cos \theta \sin \theta \theta^{\prime} \partial_{x} .
\end{aligned}
$$

Setting $\xi=\partial_{y}+\partial_{z}$, it follows that $\widetilde{\nabla}_{X} \xi=-\theta^{\prime}(x)(X \times \xi)$ for any tangent vector $X$, where $\times$ stands for the cross product associated with the orientation given by the chart $(x, y, z)$. Moreover, the scalar curvature of the manifold is $\left(\theta^{\prime}\right)^{2}-4 \cot (2 \theta) \theta^{\prime \prime}$.

Our second main result in this section decribes the totally geodesic surfaces in $M$ in case (3) in Theorem 2, It characterizes in particular the flat and spherical metrics. It can be compared to a result of E. Cartan (see [2, p. 233]). In the three-dimensional case, Cartan's theorem asserts that a three-dimensional Riemannian manifold with a totally geodesic surface passing through any point with any specified plane as tangent plane must be a space form. When the manifold admits a unit Killing 
field, our result says that the existence of very few totally geodesic surfaces suffices to characterize the space forms of non-negative curvature.

Theorem 3. Let $M$ be a Riemannian three-manifold carrying a unit Killing field $\xi$ and let $p \in M$. Suppose there is a totally geodesic surface $\Sigma_{1}$ passing through $p$ which is neither orthogonal nor tangent to $\xi$ at $p$. Then

(1) There is a second totally geodesic surface passing through $p$ which is orthogonal to $\Sigma_{1}$.

(2) If there exists a third totally geodesic surface through $p$ which is not tangent to $\xi$ at $p$, then $M$ has constant non-negative sectional curvature in a neighborhood of $p$ and thus is, around $p$, isometric to an open subset of the sphere $\mathbb{S}^{3}$ with a metric of constant curvature or to an open set of the Euclidean space $\mathbb{R}^{3}$.

(3) If $M$ does not have constant positive curvature near $p$, then there exists a totally geodesic surface through $p$ which is tangent to $\xi$ at $p$ if and only if $\tau(p)=0$.

Proof. From case (3) in Theorem 2 we can find local coordinates $(x, y, z)$ in a neighborhood $W$ of $p$ where the metric takes the form (8), the point $p$ corresponding to the origin. Restricting $\Sigma_{1}$ if necessary, we can assume that $\Sigma_{1}$ is given by the equation $z=0$.

(1) From the above local expression (8) for the metric we see that the surface $\Sigma_{0}$, defined by the equation $y=0$, is totally geodesic and is orthogonal to $\Sigma_{1}$.

(2) Suppose there is a third totally geodesic surface $\Sigma_{2}$ containing $p$ which is not tangent to $\xi$ at $p$. We first treat the case when $\Sigma_{2}$ is not orthogonal to $\xi$ at $p$. We will show that the function $\tau$ is constant in a neighborhood of $p$. This will conclude the proof as this means that, in the coordinates introduced above, $\theta(x)=\alpha x+\beta$ for some constants $\alpha$ and $\beta$. It then follows immediately from Proposition 4 that if $\alpha=0$, the metric $g$ is flat and if $\alpha \neq 0, g$ has constant sectional curvature $\alpha^{2}$.

We still denote by $\Sigma_{2}$ the component of $W \cap \Sigma_{2}$ containing $p$. Restricting $W$ and replacing $\Sigma_{2}$ by an open subset of it if necessary, we can assume the intersection $\Sigma_{1} \cap \Sigma_{2}$ is connected. For $i=1,2$, denote by $N_{i}$ a unit normal to $\Sigma_{i}$. As before we introduce the vector field $T_{i}$ tangent to $\Sigma_{i}$ and the real-valued function $\nu_{i}$ on $\Sigma_{i}$ by the orthogonal decomposition

$$
\xi=T_{i}+\nu_{i} N_{i} .
$$

We again use the same notation to denote the extensions of $N_{i}, T_{i}$ and $\nu_{i}$ to $W$ using the flow of $\xi$. Note that along $\Sigma_{1} \cap \Sigma_{2}$ the vectors $N_{1}$ and $N_{2}$ are independent, so, up to restricting $W$ if necessary, we can assume that their extensions are also pointwise independent. In the same way, as $\Sigma_{1}$ and $\Sigma_{2}$ are distinct, $T_{1}$ and $T_{2}$ are independent along $\Sigma_{1} \cap \Sigma_{2}$ and we can assume their extensions are independent in $W$.

Suppose that $\xi, T_{1}$ and $T_{2}$ are linearly independent in an open set $U \subset W$. It follows from Proposition 4 and Remark 5 that $\tau$ does not depend on $T_{1}$, that is, $T_{1}(\tau)=0$. In the same way $T_{2}(\tau)=0$. As $\xi(\tau)=0$ (see Lemma 2), we conclude that $\operatorname{grad} \tau=0$ in $U$, where grad denotes the gradient on $M$.

Suppose now that $\xi, T_{1}$ and $T_{2}$ are (pointwise) linearly dependent in some connected open set $V \subset W$. Let $S$ denote the surface tangent to the distribution spanned by $\xi$ and $T_{1}$ which passes through $p$. From the expression of the metric (8) obtained in Theorem 2 using the surface $\Sigma_{1}$, we see that the coordinate $x$ is the signed distance function to the surface $S$. As we are assuming $\xi, T_{1}$ and $T_{2}$ are 
dependent in $V$, we conclude that we obtain the same coordinate function $x$ when we use $\Sigma_{2}$ in Theorem 2. The Killing fields $T_{1}$ and $T_{2}$ are tangent to the integral surfaces of the distribution spanned by $\xi$ and $T_{1}$, that is, the level surfaces of the coordinate function $x$, and so are Killing fields on each of them. Moreover their norms depend only on $x$ (see Remark 5 ). For each $x$, the level surface corresponding to $x$ is flat, that is, locally Euclidean. The Killing fields $\xi, T_{1}$ and $T_{2}$ on such a surface correspond to constant fields under an isometry with an open subset of the Euclidean plane since they have constant norms. Therefore we have in $V$ a relation of the form

$$
\xi=\alpha_{1}(x) T_{1}+\alpha_{2}(x) T_{2} .
$$

We next show that the functions $\alpha_{1}$ and $\alpha_{2}$ are actually constant.

As $\xi, T_{1}$ and $T_{2}$ are Killing fields, we have for any vector field $Y$,

$$
\alpha_{1}^{\prime}(x)\left\langle T_{1}, Y\right\rangle+\alpha_{2}^{\prime}(x)\left\langle T_{2}, Y\right\rangle+Y\left(\alpha_{1}\right)\left\langle T_{1}, \partial_{x}\right\rangle+Y\left(\alpha_{2}\right)\left\langle T_{2}, \partial_{x}\right\rangle=0 .
$$

Taking successively $Y=T_{1}$ and $Y=T_{2}$ we get

$$
\left\langle\alpha_{1}^{\prime}(x) T_{1}+\alpha_{2}^{\prime}(x) T_{2}, T_{1}\right\rangle=\left\langle\alpha_{1}^{\prime}(x) T_{1}+\alpha_{2}^{\prime}(x) T_{2}, T_{2}\right\rangle=0 .
$$

Since $T_{1}$ and $T_{2}$ are independent, we conclude that $\alpha_{1}^{\prime}(x)=\alpha_{2}^{\prime}(x)=0$, that is, $\alpha_{1}$ and $\alpha_{2}$ are constants.

Replacing $T_{i}$ by $\xi-\nu_{i} N_{i}$, for $i=1,2$, in the decomposition $\xi=\alpha_{1} T_{1}+\alpha_{2} T_{2}$ we get

$$
\xi=\gamma_{1} \nu_{1} N_{1}+\gamma_{2} \nu_{2} N_{2}
$$

where $\gamma_{i}=\alpha_{i} /\left(\alpha_{1}+\alpha_{2}-1\right), i=1,2$. We have

$$
\widetilde{\nabla}_{\partial_{x}} \xi=\gamma_{1} \nu_{1}^{\prime}(x) N_{1}+\gamma_{2} \nu_{2}^{\prime}(x) N_{2} .
$$

As $\nu_{i}=\cos \theta_{i}(x)$, we have $\nu_{i}^{\prime}(x)=-\theta_{i}^{\prime}(x) \sin \theta_{i}(x)=\tau \sin \theta_{i}(x), i=1,2$. So

$$
\widetilde{\nabla}_{\partial_{x}} \xi=\tau\left\{\gamma_{1} \sin \theta_{1}(x) N_{1}+\gamma_{2} \sin \theta_{2}(x) N_{2}\right\} .
$$

Taking the inner product of both sides with $\xi$ we obtain

$$
\tau\left\{\gamma_{1} \sin \theta_{1}(x) \nu_{1}+\gamma_{2} \sin \theta_{2}(x) \nu_{2}\right\}=0 .
$$

That is,

$$
\tau\left\{\gamma_{1} \sin 2 \theta_{1}(x)+\gamma_{2} \sin 2 \theta_{2}(x)\right\}=0
$$

Suppose $\tau$ does not vanish in some open set $V_{0} \subset V$. Then on $V_{0}$,

$$
\gamma_{1} \sin 2 \theta_{1}(x)+\gamma_{2} \sin 2 \theta_{2}(x)=0 .
$$

Taking the derivative we get

$$
\gamma_{1} \cos 2 \theta_{1}(x)+\gamma_{2} \cos 2 \theta_{2}(x)=0 .
$$

By (9), $\left(\gamma_{1}, \gamma_{2}\right) \neq(0,0)$, so the determinant of the system in the unknowns $\gamma_{1}$ and $\gamma_{2}$ formed by equations (10) and (11) has to vanish, that is,

$$
\sin 2\left(\theta_{2}(x)-\theta_{1}(x)\right)=0
$$

However, the quantity $\theta_{2}(x)-\theta_{1}(x)$, which is a constant since $\theta_{1}^{\prime}(x)=-\tau=\theta_{2}^{\prime}(x)$, is neither equal to 0 nor to $\pm \pi / 2$. Indeed, otherwise this would imply that $\Sigma_{2}$ locally coincides with $\Sigma_{1}$ or $\Sigma_{0}$, but this contradicts the assumption that $\Sigma_{2}$ is a totally geodesic surface through $p$, different from $\Sigma_{0}$ and $\Sigma_{1}$. Consequently $\tau \equiv 0$ in $V$. 
Summarizing we have shown that $\operatorname{grad} \tau=0$ on an open dense set in a neighborhood of $p$ in $M^{3}$. Therefore $\tau$ is constant near $p$. This concludes the proof of (2) when $\Sigma_{2}$ is not orthogonal to $\xi$ at $p$.

Now suppose that $\Sigma_{2}$ is orthogonal to $\xi$ at $p$. Let $S_{0} \subset \Sigma_{2}$ denote the subset where $\Sigma_{2}$ is not orthogonal to $\xi$. By the above argument, the function $\tau$ is locally constant on $S_{0}$. Now suppose that $\Sigma_{2}$ is orthogonal to $\xi$ in an open set $S_{1} \subset \Sigma_{2}$. So $\xi$ is the unit normal to $\Sigma_{2}$ on $S_{1}$. As $\Sigma_{2}$ is totally geodesic, by the formula in Lemma 2, we have $\tau \equiv 0$ on $S_{1}$. Consequently, denoting by $\operatorname{grad}^{\Sigma_{2}}$ the gradient on $\Sigma_{2}$, we have $\operatorname{grad}^{\Sigma_{2}} \tau=0$ on an open dense subset of $\Sigma_{2}$ and so $\tau$ is constant on $\Sigma_{2}$. As $\xi$ is transversal to $\Sigma_{2}$ and $\xi(\tau)=0$, we conclude again that $\tau$ is constant in a neighborhood of $p$ in $M^{3}$. This concludes the proof of (2).

(3) Suppose $\tau(p)=0$, that is, $\theta^{\prime}(0)=0$. Then from Proposition 4 the surface given by $x=0$ is totally geodesic. Conversely, suppose there is a connected totally geodesic surface $\Sigma$ through $p$ which is tangent to $\xi$ at $p$. We may assume $\Sigma$ is contained in the coordinate neighborhood where the metric on $M$ takes the form (8). By the same arguments as in (2), we get that $\tau$ is constant on any connected open subset of $\Sigma$ where $\xi$ is not tangent to $\Sigma$. Moreover $\tau$ vanishes on any open subset of $\Sigma$ where $\xi$ is tangent to $\Sigma$ (see the proof of (2) in Theorem 2). As previously, we conclude that $\tau$ is constant on $\Sigma$.

Denote by $\pi$ the projection on the $x$-axis. We consider three cases:

- First case: $I:=\pi(\Sigma)$ contains an open interval containing 0 . It follows that $\tau$ (which depends only on $x$ ) is constant in a neighborhood of $p$. So, near $p, M$ is flat or has constant positive curvature. The second possibility is excluded by hypothesis and consequently $\tau$ is identically zero near $p$.

- Second case: $I=\{0\}$, that is, $\Sigma \subset\{x=0\}$. From the equations in Proposition 4. we see that the surface $\{x=0\}$ is totally geodesic if and only if $\theta^{\prime}(0)=0$, that is, if and only if $\tau(p)=0$.

- Third case: $\pi(\Sigma) \subset[0,+\infty)$ or $\pi(\Sigma) \subset(-\infty, 0]$. This means the surface $\Sigma$ is on one side of the surface $\{x=0\}$. The extrinsic curvature of the surface $\{x=0\}$ is $K_{\text {ext }}=-\left(\theta^{\prime}(0)\right)^{2}=-\tau(p)^{2}$ as is seen from Proposition 4. It is therefore a saddle surface if $\tau(p) \neq 0$ and we are led in this case to a contradiction since it is a general fact that a totally geodesic surface tangent to a saddle surface at a point cannot lie on one side of it near the tangency point. So necessarily $\tau(p)=0$.

\section{Properties of the three-spaces}

In this section we shall discuss some properties of three-dimensional spaces $M^{3}$ with a unit Killing field $\xi$ that admit totally geodesic surfaces which are neither orthogonal nor tangent to $\xi$. From Theorem 2 we know that such a manifold locally admits a metric of type

$$
g=d x^{2}+\sin ^{2} \theta(x) d y^{2}+\cos ^{2} \theta(x) d z^{2},
$$

with $\xi=\partial_{y}+\partial_{z}$.

The following result, which can be checked through straightforward computations, states that these three-spaces admit Riemannian submersions onto a surface with a Killing field. 
Proposition 5. Given $M^{3}$ as above, consider a surface $M^{2}$ with local coordinates $(u, v)$ and metric

$$
d u^{2}+\frac{1}{4} \sin ^{2}(2 \theta(u)) d v^{2} .
$$

Then the mapping $\pi: M^{3} \rightarrow M^{2}:(x, y, z) \mapsto(u, v)=(x, y-z)$ is a Riemannian submersion whose fibers are integral curves of the unit Killing field $\xi=\partial_{y}+\partial_{z}$. We remark that the Gaussian curvature of $M^{2}$ is $K=4\left(\theta^{\prime}\right)^{2}-2 \cot (2 \theta) \theta^{\prime \prime}$.

Let us now study some global properties of $M^{3}$. In particular we want to investigate which manifolds admit a smooth metric which, in local coordinates, is given by (12).

We will first recall two lemmas from 8 , on a class of more general doubly warped products

$$
\left(I \times \mathbb{S}^{p} \times \mathbb{S}^{q}, d x^{2}+\varphi^{2}(x) g_{\mathbb{S}^{p}}+\psi^{2}(x) g_{\mathbb{S} q}\right),
$$

where $I \subseteq \mathbb{R}$ is an open interval and $g_{\mathbb{S}^{p}}$ and $g_{\mathbb{S}^{q}}$ are the standard Riemannian metrics on $\mathbb{S}^{p}$ and $\mathbb{S}^{q}$

Lemma 3 ([8]). If $\varphi:(0, b) \rightarrow(0, \infty)$ is smooth and $\varphi(0)=0$, then the metric in (13) is smooth at $x=0$ if and only if $\varphi^{(e v e n)}(0)=0, \varphi^{\prime}(0)=1, \psi(0)>0$ and $\psi^{(\text {odd })}(0)=0$. In this case, the topology near $x=0$ is $\mathbb{R}^{p+1} \times \mathbb{S}^{q}$.

Lemma 4 ([8]). If $\varphi:(0, b) \rightarrow(0, \infty)$ is smooth and $\varphi(b)=0$, then the metric in (13) is smooth at $x=b$ if and only if $\varphi^{(e v e n)}(b)=0, \varphi^{\prime}(b)=-1, \psi(b)>0$ and $\psi^{(\text {odd })}(b)=0$. In this case, the topology near $x=b$ is also $\mathbb{R}^{p+1} \times \mathbb{S}^{q}$.

These results allow us to prove that a smooth metric of type (12) exists on the simply connected manifolds $\mathbb{S}^{3}, \mathbb{S}^{2} \times \mathbb{R}$ and $\mathbb{R}^{3}$.

Proposition 6. If $\theta:[0, b] \rightarrow \mathbb{R}$ is a smooth function such that $\theta^{-1}\{0\}=\{0\}$ and $\theta^{-1}\{\pi / 2\}=\{b\}$, then the metric (12) defines a smooth metric on $\mathbb{S}^{3}$ if and only if $\theta^{\prime}(0)=\theta^{\prime}(b)=1$ and $\theta^{(2 k)}(0)=\theta^{(2 k)}(b)=0$ for any positive integer $k$.

Proof. It follows from the assumptions on $\theta$ that $\theta(0, b)=(0, \pi / 2)$. Hence, the functions $\varphi=\sin \theta$ and $\psi=\cos \theta$ are strictly positive on $(0, b)$. Lemmas 3 and 4 yield that (12) then gives rise to a smooth metric on $\mathbb{S}^{3}$ if and only if the conditions of Lemma 3 are satisfied at $x=0$ and the conditions of Lemma 4 are satisfied at $x=b$, with the roles of $\varphi$ and $\psi$ interchanged.

It is easy to see that the condition $\varphi^{\prime}(0)=1$ is equivalent to $\theta^{\prime}(0)=1$ and that $\psi(0)>0$ is automatically satisfied. Similarly $\psi^{\prime}(b)=-1$ if and only if $\theta^{\prime}(b)=1$ and $\varphi(b)>0$ is automatically satisfied. The remaining conditions are thus

$$
\varphi^{(\text {even })}(0)=0, \quad \psi^{(\text {odd })}(0)=0, \quad \psi^{(\text {even })}(b)=0, \quad \varphi^{(\text {odd })}(b)=0 .
$$

After a computation and using that $\theta^{\prime}(0)=\theta^{\prime}(b)=1$, one sees that these conditions are equivalent to $\theta^{(2 k)}(0)=\theta^{(2 k)}(b)=0$ for any integer $k>0$.

Remark 6. The function $\theta(x)=x$ satisfies the conditions given in Proposition 6 , In this case, the metric (12) corresponds to the standard metric on $\mathbb{S}^{3}$ and the Riemannian submersion of Proposition 5 is the classical Hopf fibration.

Proposition 7. If $\theta:[0, b] \rightarrow[0, \infty)$ is a smooth function such that $\theta^{-1}\{0\}=\{0, b\}$ and $\theta^{-1}\{\pi / 2\}=\varnothing$, then the metric (12) defines a smooth metric on $\mathbb{S}^{2} \times \mathbb{R}$ if and only if $\theta^{\prime}(0)=-\theta^{\prime}(b)=1$ and $\theta^{(2 k)}(0)=\theta^{(2 k)}(b)=0$ for any non-negative integer $k$. 
Proof. We remark that the functions $\varphi=\sin \theta$ and $\psi=\cos \theta$ are positive on $(0, b)$. Hence, (12) defines a smooth metric on $\mathbb{S}^{2} \times \mathbb{R}$ if and only if the conditions of Lemmas 3 and 4 are satisfied. We can now proceed in an analogous way as in the proof of Proposition 6 to obtain the result.

Proposition 8. If $\theta: \mathbb{R} \rightarrow \mathbb{R}$ is a smooth function such that $\theta^{-1}\{k \pi \mid k \in \mathbb{Z}\}=$ $\theta^{-1}\{\pi / 2+k \pi \mid k \in \mathbb{Z}\}=\varnothing$, then (12) defines a smooth metric on $\mathbb{R}^{3}$, which is moreover complete.

Proof. It is clear that the metric is smooth under the given assumptions. To prove completeness, we may assume that $\theta(x) \in(0, \pi / 2)$ for all $x \in \mathbb{R}$. Now let $\gamma$ : $[0, T) \rightarrow \mathbb{R}^{3}: t \mapsto\left(\gamma_{1}(t), \gamma_{2}(t), \gamma_{3}(t)\right)$ be a curve which diverges to infinity, i.e., such that $\gamma_{1}(t)^{2}+\gamma_{2}(t)^{2}+\gamma_{3}(t)^{2}$ tends to infinity if $t$ tends to $T$. We have to prove that the length of this curve with respect to the metric (12),

$$
L(\gamma)=\int_{0}^{T} \sqrt{\left(\gamma_{1}^{\prime}(t)\right)^{2}+\sin ^{2}\left(\theta\left(\gamma_{1}(t)\right)\right)\left(\gamma_{2}^{\prime}(t)\right)^{2}+\cos ^{2}\left(\theta\left(\gamma_{1}(t)\right)\right)\left(\gamma_{3}^{\prime}(t)\right)^{2}} d t,
$$

is infinite. Therefore, we consider two cases.

First, assume that $\gamma_{1}$ is unbounded. In this case, we have

$$
L(\gamma) \geq \int_{0}^{T}\left|\gamma_{1}^{\prime}(t)\right| d t \geq \lim _{t \rightarrow T}\left|\gamma_{1}(t)-\gamma_{1}(0)\right|=\infty .
$$

Next, assume that $\gamma_{1}$ is bounded. In that case the function $\theta\left(\gamma_{1}(t)\right)$ is bounded away from 0 and $\pi / 2$ and hence there exists a real constant $c>0$ such that $\sin \left(\theta\left(\gamma_{1}(s)\right)\right) \geq c$ and $\cos \left(\theta\left(\gamma_{1}(s)\right)\right) \geq c$. This implies that

$$
L(\gamma) \geq c \int_{0}^{T} \sqrt{\left(\gamma_{2}^{\prime}(t)\right)^{2}+\left(\gamma_{3}^{\prime}(t)\right)^{2}} d t=\infty .
$$

The last equality is due to the fact that the integral appearing on the left-hand side is the Euclidean length of the projection of the curve $\gamma$ onto the $(y, z)$-plane. Since $\gamma$ diverges to infinity but $\gamma_{1}$ is bounded, this projection must have infinite length.

It is possible to check, using for instance our Theorem 3, that in the examples of Propositions 6 and 7, through the points where $x=0$ or $x=b$, there is no totally geodesic surface which is not tangent to the unit Killing field unless the function $\theta^{\prime}$ is constant in a neighborhood of $x=0$ and $x=b$, respectively. This is not a mere coincidence. We actually have the following global result.

Theorem 4. Let $M$ be a connected and simply connected complete Riemannian three-manifold carrying a unit Killing field $\xi$. Suppose that

(1) no open subset of $M$ has constant non-negative curvature,

(2) through each point of $M$ there passes a totally geodesic surface which is neither orthogonal nor tangent to $\xi$.

Then $M$ is isometric to $\mathbb{R}^{3}$ endowed with the metric,

$$
d s^{2}=d x^{2}+\sin ^{2} \theta(x) d y^{2}+\cos ^{2} \theta(x) d z^{2},
$$


where $\theta: \mathbb{R} \rightarrow(0, \pi / 2)$ is a smooth function whose derivative $\theta^{\prime}$ is not constant on any interval. Moreover $\xi=\partial_{y}+\partial_{z}$.

Proof. Let $p_{0}$ be a fixed point in $M$. By Theorems 2 and 3 , admits an orthogonal decomposition, $\xi=X_{1}+X_{2}$, in a neighborhood of $p_{0}$, where $X_{1}$ and $X_{2}$ are two Killing fields which commute and have no zeros. Moreover this decomposition is unique up to ordering of $X_{1}$ and $X_{2}$. By the same theorems, given any point $p \in M$ and any continuous path $\gamma$ joining $p_{0}$ to $p$, we can extend continuously this decomposition along $\gamma$ until the point $p$. As $M$ is simply connected, by a standard monodromy argument, the decomposition we obtain at $p$ is independent on the choice of the path $\gamma$. We get in this way a global orthogonal decomposition $\xi=X_{1}+X_{2}$, where $X_{1}$ and $X_{2}$ are now global smooth Killing vector fields on $M$ which commute and have no zeros.

Denote by $u$ a unit vector field on $M$ orthogonal to $X_{1}$ and $X_{2}$. Such a global smooth vector field exists since $M$ is orientable. From the proof of (3)(ii) of Theorem 2. we know that the distribution spanned by $u$ and $X_{1}$ is integrable and its integral surfaces are totally geodesic. Therefore the manifold $M$ admits a foliation $\mathcal{F}$ by totally geodesic surfaces. Let $\mathcal{F}^{\perp}$ be the orthogonal foliation, that is, the foliation by the orbits of $X_{2}$. By a result of Carrière and Ghys $\left[4,\left(\mathcal{F}, \mathcal{F}^{\perp}\right)\right.$ is a product. This means there is a diffeomorphism between $M$ and the product $\Sigma \times \mathbb{R}$, where $\Sigma$ is any fixed leaf of $\mathcal{F}$, sending the leaves of $\mathcal{F}$ to $\Sigma \times\{*\}$ and those of $\mathcal{F}^{\perp}$ to $\{*\} \times \mathbb{R}$. Denote by $z$ the coordinate on the $\mathbb{R}$ factor. Under this diffeomorphism, the vector field $X_{2}$ therefore corresponds to the field $f(z) \partial_{z}$ for some function $f$. So, up to reparametrizing the $\mathbb{R}$ factor, we can assume that $X_{2}$ corresponds to the field $\partial_{z}$. It is clear that $\Sigma$ is simply connected and is therefore, topologically, either a plane or a sphere. $X_{1}$ is vector field on $\Sigma$ which has no zeros, so $\Sigma$ is topologically a plane. It is moreover not difficult to check that $\Sigma$ is complete.

Fix an orientation on $\Sigma$ and denote by $J$ the rotation over 90 degrees in $T \Sigma$. As in the proof of (3) of Theorem 2, we consider on $\Sigma$ the fields $X_{1}$ and $J X_{1}$ which commute, $\left[X_{1}, J X_{1}\right]=0$, and are complete since they have bounded norms and $\Sigma$ is complete. It follows that we can find a global chart for $\Sigma$ with domain $\mathbb{R}^{2}$ and $\partial_{x}=J X_{1}$ and $\partial_{y}=X_{1}$ for the standard coordinates $(x, y)$ on $\mathbb{R}^{2}$. We include a proof of this fact for completeness. Let $\left(\phi_{x}\right)_{x \in \mathbb{R}}$ and $\left(\psi_{y}\right)_{y \in \mathbb{R}}$ be the flows of $J X_{1}$ and $X_{1}$, respectively. Consider the mapping,

$$
(x, y) \in \mathbb{R}^{2} \rightarrow F(x, y)=\left(\phi_{x} \circ \psi_{y}\right)\left(p_{0}\right) \in \Sigma .
$$

$F$ is a local diffeomorphism with $(d F)\left(\partial_{x}\right)=J X_{1}$ and $(d F)\left(\partial_{y}\right)=X_{1}$. We will show it is a global diffeomorphism and this will provide the global chart we want.

- $F$ is one-to-one: By contradiction, suppose $\left(x_{1}, y_{1}\right)$ and $\left(x_{2}, y_{2}\right)$ are distinct points in $\mathbb{R}^{2}$ with $F\left(x_{1}, y_{1}\right)=F\left(x_{2}, y_{2}\right)$. Assume that $x_{1}=x_{2}$ and $y_{1} \neq y_{2}$, then the orbit of $X_{1}$ through $\phi_{x_{1}}\left(p_{0}\right)$ will be closed and will bound a disk in $\Sigma$ inside which necessarily $X_{1}$ will have a zero, which is a contradiction. The case $x_{1} \neq x_{2}$ and $y_{1}=y_{2}$ is similar. Assume now that $x_{1} \neq x_{2}$ and $y_{1} \neq y_{2}$. Set $p_{1}=\left(\phi_{x_{1}} \circ \psi_{y_{1}}\right)\left(p_{0}\right)=\left(\phi_{x_{2}} \circ \psi_{y_{2}}\right)\left(p_{0}\right)$. The orbit of $J X_{1}$ through $p_{0}$ and the orbit of $X_{1}$ through $p_{1}$ intersect at two distinct points, namely $\phi_{x_{1}}\left(p_{0}\right)$ and $\phi_{x_{2}}\left(p_{0}\right)$. However an orbit of $J X_{1}$ can intersect an orbit of $X_{1}$ at most once. Indeed let $\gamma_{1}$ and $\gamma_{2}$ be orbits of $X_{1}$ and $J X_{1}$, respectively. Assume they intersect more than once. Then there will be a bounded disk $\Omega$ in $\Sigma$ with boundary the union of an $\operatorname{arc} \alpha_{1} \subset \gamma_{1}$ and an arc $\alpha_{2} \subset \gamma_{2}$ with common endpoints $p$ and $q$. We assume that 
along $\alpha_{2}$, the field $X_{1}$ points into $\Omega$. The case when $X_{1}$ points outside $\Omega$ can be treated in a similar way. Consider any point $q_{1}$ on $\alpha_{2}$ distinct from $p$ and $q$. The positive semi-orbit $\beta:=\left\{\psi_{t}\left(q_{1}\right), t>0\right\}$ of $X_{1}$ through $q_{1}$ will be entirely contained in $\Omega$. It follows from the Poincaré-Bendixon theorem (see for instance [5]) that the accumulation set of $\beta$ must contain a zero or a closed orbit of $X_{1}$, which is again a contradiction.

- $F$ is onto: Since $F$ is a local diffeomorphism, the image $F\left(\mathbb{R}^{2}\right)$ is open in $\Sigma$, so to conclude it is enough to see that it is closed. Let $\left(x_{n}, y_{n}\right), n \in \mathbb{N}$, be a sequence of points in $\mathbb{R}^{2}$ with $F\left(x_{n}, y_{n}\right) \rightarrow p_{\infty} \in \Sigma$ as $n \rightarrow \infty$. For $\epsilon>0$ small enough, the mapping $(x, y) \in(-\epsilon, \epsilon) \times(-\epsilon, \epsilon) \rightarrow\left(\phi_{x} \circ \psi_{y}\right)\left(p_{\infty}\right) \in \Sigma$ is an embedding with image an open neighborhood $V$ of $p_{\infty}$. For $n$ fixed and big enough, we have $F\left(x_{n}, y_{n}\right) \in V$ and so there is $(x, y) \in(-\epsilon, \epsilon) \times(-\epsilon, \epsilon)$ such that $\left(\phi_{x} \circ \psi_{y}\right)\left(p_{\infty}\right)=\left(\phi_{x_{n}} \circ \psi_{y_{n}}\right)\left(p_{0}\right)$. Therefore $p_{\infty}=\phi_{x_{n}-x} \circ \psi_{y_{n}-y}\left(p_{0}\right)$ lies in $F\left(\mathbb{R}^{2}\right)$.

In the global coordinates $(x, y, z)$, as in the proof of (3) in Theorem 2 and with the same notation, the metric on $M$ writes

$$
d s^{2}=\left(1-\nu(x)^{2}\right)\left(d x^{2}+d y^{2}\right)+\nu(x)^{2} d z^{2},
$$

where $\nu(x)=\left\|X_{2}\right\|^{2}$ is a function of $x$ alone. We now make the change of coordinate $\bar{x}(x)=\int \sqrt{1-\nu(x)^{2}} d x$. By the completeness of the metric $g$, the function $\bar{x}$ is a bijection from $\mathbb{R}$ onto $\mathbb{R}$. Setting $\nu(x)=\cos \theta(\bar{x})$ for some smooth function $\theta: \mathbb{R} \rightarrow$ $(0, \pi / 2)$, the metric writes in the global coordinates $(\bar{x}, y, z)$

$$
d s^{2}=d \bar{x}^{2}+\sin ^{2} \theta(\bar{x}) d y^{2}+\cos ^{2} \theta(\bar{x}) d z^{2} .
$$

Condition (1) in the statement means precisely that $\theta^{\prime}$ is not constant on any interval (see the proof of (2) in Theorem 3 ).

\section{REFERENCES}

[1] V. N. Berestovskii and Yu. G. Nikonorov, Killing vector fields of constant length on Riemannian manifolds (Russian), Sibirsk. Mat. Zh. 49 (2008), 497-514, translation in Sib. Math. J. 49 (2008), 395-407. MR2442533 (2009f:53046)

[2] M. Berger, A panoramic view of Riemannian geometry, Springer-Verlag, Berlin, 2003. MR.2002701 (2004h:53001)

[3] G. Calvaruso, D. Kowalczyk, and J. Van der Veken, On extrinsically symmetric hypersurfaces of $\mathbb{H}^{n} \times \mathbb{R}$, Bull. Austral. Math. Soc. 82 (2010), 390-400. MR2737951 (2012a:53088)

[4] Y. Carrière and E. Ghys, Feuilletages totalement géodésiques, Acad. Brasil. Ciênc. 53 (1981), no. 3, 427-432. MR663239 (83m:57019)

[5] M. Hirsch and S. Smale, Differential equations, dynamical systems, and linear algebra, Pure and Applied Mathematics, Vol. 60. Academic Press [A subsidiary of Harcourt Brace Jovanovich, Publishers], New York-London, 1974. MR0486784 (58:6484)

[6] S. Montiel, Unicity of constant mean curvature hypersurfaces in some Riemannian manifolds, Indiana Univ. Math. J. 48 (1999), no. 2, 711-748. MR.1722814 (2001f:53131)

[7] A. Pauly, Flachen mit lauter Nabelpunkten, Elem. Math. 63 (2008), no. 3, 141-144. MR2424898 (2009d:53007)

[8] P. Petersen, Riemannian Geometry, Graduate Texts in Mathematics, Springer-Verlag, New York, 1998. MR:1480173 (98m:53001)

[9] A. Ros and F. Urbano, Lagrangian submanifolds of $\mathbb{C}^{n}$ with conformal Maslov form and the Whitney sphere, J. Math. Soc. Japan 50 (1998), no. 1, 205-226. MR.1484619 (98k:53081)

[10] R. Souam and E. Toubiana, On the classification and regularity of umbilic surfaces in homogeneous 3-manifolds. XIV School on Differential Geometry, Mat. Contemp. 30 (2006), 201-215. MR2373511(2009f:53080)

[11] R. Souam and E. Toubiana, Totally umbilic surfaces in homogeneous 3-manifolds, Comment. Math. Helv. 84 (2009), 673-704. MR 2507258(2010f:53079) 
[12] J. Van der Veken, Higher order parallel surfaces in Bianchi-Cartan-Vranceanu spaces, Result. Math. 51 (2008), 339-359. MR2400172 (2009h:53128)

[13] J. Van der Veken and L. Vrancken, Parallel and semi-parallel hypersurfaces of $\mathbb{S}^{n} \times \mathbb{R}$, Bull. Braz. Math. Soc. N. S. 39 (2008), 355-370. MR2473852(2009k:53136)

Institut de Mathématiques de Jussieu, CNRS UMR 7586, Université Paris Diderot, Paris 7, "Géométrie et Dynamique", Site Chevaleret, Case 7012, 75205, Paris Cedex 13, FRANCE

E-mail address: souam@math.jussieu.fr

Departement Wiskunde, Katholieke Universiteit Leuven, Celestijnenlaan 200 B, Box 2400, BE-3001 Leuven, Belgium

E-mail address: joeri.vanderveken@wis.kuleuven.be 